Reprod. Nutr. Dévelop., 1988, 28 (6 B), 1541-1554.

\title{
L'action du génome paternel au début du développement embryonnaire
}

\author{
J. P. RENARD, Jacqueline BARRA, C. BABINET
}

Unité de Génétique des Mammifères,

Institut Pasteur, 25, rue du Dr-Roux, 75015 Paris.

\section{Summary. Action of the paternal genome during early embryonic development.}

Recent experiments using nuclear transfer between mouse embryos shows that the parental genomes are not equivalent : both of them must be present at least in a part of the blastomeres to allow full term development of the embryo. The paternal genome is preferentially involved in the development of trophoectodermic cells and appears to be eliminated from the primitive ectoderm when kept alone in androgenetic cells aggregated with normal ones. Differences of expression between parental genomes affect not only the $X$ chromosome, but also several autosomes as evidenced by genetic analysis using meiotic non-disjunction. The nature of the imprinting mechanism that leads to the modification of parental homologous chromosomes is unknown. From experiments involving a particular strain of mice called DDK it appears that the egg cytoplasm may exert a specific action not only on the male pronucleus at the one-cell stage, but also on the paternal contribution of the diploid nucleus of the embryo at the 2-cell stage ; a paternal developmental effect on the cytoplasm of one-cell stage embryo can also be demonstrated. These results show that nucleocytoplasmic interactions regulate early paternal gene expression differently from the maternal one.

\section{Introduction.}

Le rôle du génome mâle dans le développement de l'embryon de mammifère, est le plus souvent considéré sous deux aspects: son implication dans les événements liés à la fécondation, et sa participation à la restauration de l'état diploïde après réunion avec le génome de l'ovocyte.

Chez plusieurs espèces, notamment les espèces domestiques où l'on a largement recours à l'insémination artificielle, il a pu être montré que le taux de mortalité embryonnaire (estimé à partir du taux de femelles qui ne reviennent pas en cycle) pouvait varier de plus de $30 \%$ selon le mâle utilisé (Bearden et al., 1956). Il est également bien établi que le mâle peut être directement responsable des arrêts de développement dus à l'existence d'anomalies génétiques (Gropp et Winking, 1981 ; Gustavsson, 1979 ; Mattei, 1984).

Ces données tendent à définir le rôle du génome paternel à partir de la qualité physiologique du gamète mâle au moment de la fécondation, et de sa qualité génétique héritée des deux parents (Thibault, 1985). Elles ne prennent pas en 
compte le fait, récemment mis à jour, que chaque génome parental a une empreinte particulière ("imprinting »). Ce marquage fait que la présence, à la fois d'un génome maternel et d'un génome paternel est nécessaire à la réalisation d'une embryogenèse normale (Surani et al., 1984; McGrath et Solter, 1984a ; Surani, 1986).

Le but de cet article est de présenter les données récentes obtenues principalement chez la souris, qui mettent en évidence l'action propre au génome paternel au cours du développement embryonnaire.

\section{Nécessité d'un génome paternel pour un développement normal.}

L'absence de participation du gamète mâle au développement embryonnaire, ou parthénogenèse, est un mode de reproduction que l'on rencontre dans la plupart des groupes animaux et qui survient surtout dans des conditions écologiques particulières (Cuellar, 1977). Il résulte de l'activation spontanée de l'ovocyte, qui se développe pour donner un nouvel individu. C'est un phénomène qui peut se produire dans toutes les classes de vertébrés avec une exception notable, la classe des mammifères.

Des méthodes ont été développées pour produire expérimentalement des embryons de mammifères parthénogénétiques en les activant à l'aide d'agents physiques ou chimiques (Kaufman, 1983). Ces embryons se divisent apparemment normalement in vitro, et après transfert s'implantent. Mais leur développement s'arrête au plus tard à la mi-gestation (fig. 1).

Jusqu'à une date récente, 2 hypothèses étaient avancées pour expliquer l'absence de développement à terme des embryons parthénotes. La première considérait que des gènes récessifs léthaux s'exprimaient dans ces embryons homozygotes; la seconde supposait que des facteurs extragénétiques d'origine paternelle, nécessaires au développement normal, étaient apportés par le spermatozoïde au moment de la fécondation, et que leur absence chez les parthénotes était responsable de l'arrêt du développement.

La mise au point d'une technique d'énucléation qui permet d'obtenir des noyaux intacts à l'intérieur de petites vésicules entourées de membrane plasmique (karyoplastes) a été une étape décisive pour l'étude de la contribution respective des génomes paternel et maternel de l'embryon de souris (McGrath et Solter, 1983). Cette technique a permis, au cours des trois dernières années, de réaliser des expériences d'échange de noyaux (transfert nucléaire) entre différents types d'embryons et d'établir de manière définitive qu'il faut hériter à la fois d'un génome de chacun des parents pour que le développement de l'embryon de souris se déroule normalement jusqu'à terme.

L'hypothèse de l'expression de gènes récessifs léthaux s'avéra être incompatible avec le fait que si, au stade 1 cellule, on échange entre des embryons fécondés de 2 lignées différentes, les pronoyaux paternels et maternels (donc si on reconstruit des génomes diploïdes hétérozygotes), on obtient un développement à terme uniquement en associant un génome de chaque type ; dans les autres cas, c'est-à-dire quand on associe 2 génomes paternels ou 2 génomes maternels, aucune naissance n'est obtenue (McGrath et Solter, 1984a). 


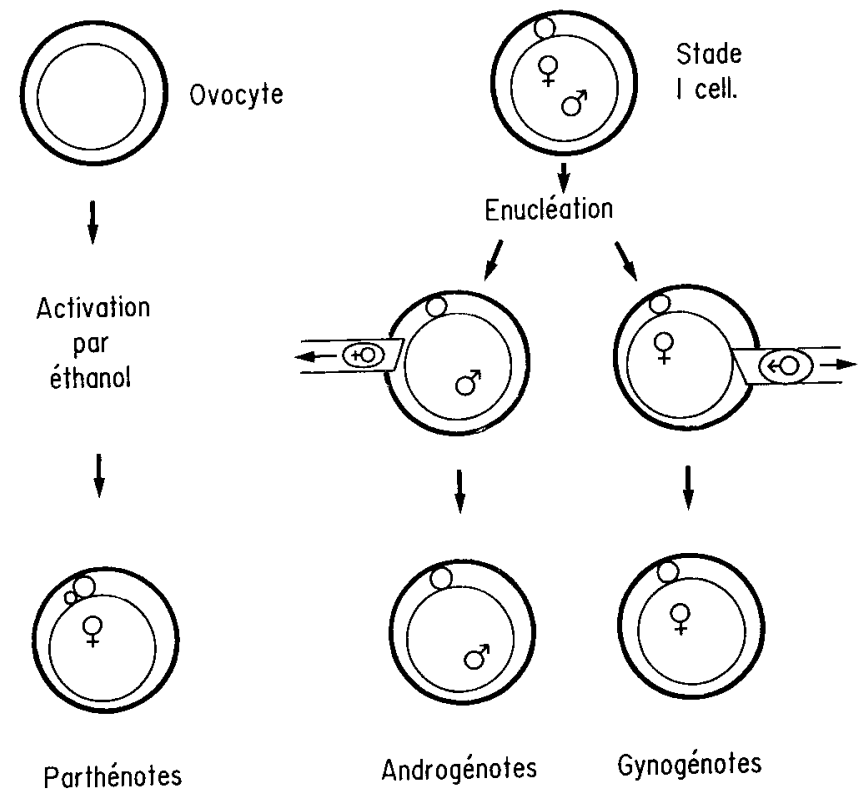

FIG. 1. - Méthodes d'obtention d'embryons uniparentaux.

L'hypothèse de l'existence de facteurs extragénétiques dut également être abandonnée quand il fut établi que le cytoplasme d'un embryon parthénote (énuclée) peut assurer le développement à terme d'embryons reconstitués avec les deux pronoyaux provenant d'un œuf normalement fécondé, et qu'inversement les noyaux d'un œuf parthénote placés dans le cytoplasme d'un œuf fécondé puis énuclée, ne permettent pas d'obtenir un développement à terme (Mann et Lovell-Badge, 1984).

Ces expériences montrent que les 2 pronuclei ne sont pas équivalents, ceci bien que le génome soit identique (au chromosome $Y$ près) dans les deux sexes, au moins dans les lignées consanguines. L'absence d'un génome paternel empêche les embryons parthénotes de souris (et probablement ceux des mammifères) de se développer jusqu'à terme.

\section{La contribution du génome paternel au début du développement embryonnaire.}

$\mathrm{Si}$, pendant les premières heures qui suivent la fécondation, on retire par micromanipulation le pronoyau femelle d'un œuf fécondé, on obtient un embryon dont le développement ne dépend plus que du seul génome apporté par le spermatozoïde. De tels embryons, dits androgénotes, sont capables de se diviser, mais leurs possibilités de développement sont moins grandes que celles des 
embryons gynogénotes ; contrairement à ces derniers, ils ne peuvent, in vitro, atteindre le stade blastocyste, et seulement $5 \%$ d'entre eux, contre plus de $30 \%$ pour les embryons gynogénotes, se divisent jusqu'au stade 4 à 8 cellules (Surani et al., 1986). En substituant au pronoyau femelle de l'œuf un $2^{\mathrm{e}}$ pronoyau mâle, on obtient un androgénote diploïde qui peut atteindre le stade blastocyste. Mais même en tenant compte du fait que le quart des embryons ainsi formés sont de constitution $Y Y$, et ne se développent pas, il apparaît que les possibilités de développement des androgénotes diploïdes restent plus faibles que celles des parthénotes ou gynogénotes diploïdes: après 5 jours in vitro, les taux de blastocystes formés sont respectivement de $10 \%$ et $60 \%$ (Barton et al., 1984 ; McGrath et Solter, 1984a).

Les faibles potentialités de développement que manifestent les embryons androgénotes ne résultent pas de possibilités réduites de multiplication de leurs blastomères. En effet, si au stade 4 cellules on agrège des blastomères androgénotes à des blastomères de même stade provenant d'embryons normaux, on obtient à la mi-gestation des fœtus dont les annexes embryonnaires, sont pour partie (jusqu'à près de la moitié dans le trophoblaste) constituées par des cellules androgénotes. Mais celles-ci sont pratiquement absentes des tissus qui forment le fœtus proprement dit : celui-ci dérive des blastomères provenant des embryons normaux (Surani et al., 1988).

De même, si on transplante des embryons androgénotes, le développement du fœtus est très réduit ; il ne se développe pas au-delà du stade 6 à 8 somites, et les sites d'implantation contiennent surtout des tissus semblables aux annexes embryonnaires (Surani, 1986). Une situation analogue est observée chez l'homme avec la formation de môles hydatiformes qui résultent du développement d'embryons dont le génome femelle n'a pu devenir fonctionnel (Szulman et Surti, 1984).

En outre, il apparaît que la contribution au développement des cellules androgénotes est très différente de celle des cellules parthénotes (ou gynogénotes) qui n'ont reçu qu'un génome maternel. Ces dernières en effet, agrégées avec des blastomères normaux, donnent des individus chimériques, et peuvent participer à la formation de tous les tissus y compris ceux de la lignée germinale (Surani et al., 1977 ; Stevens et al., 1977 ; Stevens, 1978 ; Anderegg et Markert, 1987). Par contre, elles sont très peu présentes dans le trophoblaste et l'endoderme primitif (Surani et al., 1987 ; Nagy et al., 1987) : leur évolution est donc différente de celle des blastomères androgénotes. Ceux-ci sont éliminés de l'ectoderme primitif d'où dérive le fœutus et leur différenciation préférentielle vers les tissus extra-embryonnaires traduit l'existence de défauts d'expression du génome quand s'établit la lignée ectodermique.

Les phénotypes en apparence opposés que confèrent aux blastomères chacun des génomes parentaux ne sont toutefois pas complémentaires : l'agrégation d'embryons parthénotes avec des embryons androgénotes (Surani et al., 1987) ne permet pas la naissance de jeunes viables : à 10 jours de gestation, on obtient des fœtus formés essentiellement à partir de tissus dérivés de blastomères parthénotes, alors que les membranes extraembryonnaires dérivent des blasto- 
mères androgénotes; mais ces embryons composites sont résorbés vers le $12^{\mathrm{e}}$ jour.

On ne sait pas aujourd'hui quel est dans un embryon chimère, le nombre minimum de blastomères normaux qu'il suffirait d'associer à des blastomères androgénotes pour permettre l'obtention de jeunes viables. On ne sait pas non plus à partir de quand au cours du développement, la présence de cellules possédant à la fois les 2 génomes parentaux est nécessaire pour assurer un développement normal.

Chez les mammifères, la mise en activité du génome de l'embryon se produit après une courte période de développement dominée par l'utilisation des transcrits maternels présents dans l'ovocyte avant la fécondation. Ce moment est maintenant bien connu chez plusieurs espèces : il se situe vers la fin du stade 2 cellules chez la souris (Bensaude et al., 1983) et à partir du stade 8 cellules chez l'homme (Braude et al., 1988). Or on a récemment montré que des embryons diploïdes formés par la fusion, au stade 2 cellules, d'un blastomère issu d'embryons androgénotes avec un blastomère issu d'embryons parthénotes, pouvaient donner naissance à des jeunes viables (Barra et Renard, 1988) : le génome paternel peut donc être mis en activité dans un environnement cytoplasmique propre, puis après avoir été réuni avec le génome maternel, contribuer au développement à terme.

Les interactions entre le génome paternel et le génome maternel ne sont donc pas nécessaires avant le stade 2 cellules. A partir de quand le génome paternel exerce-t-il une contribution spécifique dans le développement embryonnaire?

\section{Expression du génome paternel au début du développement embryon- naire.}

Grâce à l'existence de variants génétiques exprimant deux formes différentes d'une même protéine, repérables par une simple électrophorèse, on peut distinguer l'activité des allèles de chaque contribution parentale. En utilisant cette approche avec des variants de la $\beta^{2}$-microglobuline, un peptide associé aux produits des gènes majeurs d'histocompatibilité, on a pu mettre en évidence dès le stade 2 cellules chez la souris, l'existence d'une activité de la composante paternelle du génome (Sawicki et al., 1981). Cette expression est donc précoce puisqu'elle se produit dès que s'initient les synthèses embryonnaires. Pour d'autres gènes comme la $\beta$-glucuronidase (Wudl et Chapman, 1976), ou la glucose phosphate isomérase 1 (GPI-1, Brinster, 1973), la composante paternelle s'exprime au cours des premières divisions de segmentation, respectivement aux stades 4 et 8 cellules. Dans le cas de la GPI-1, on a montré que l'expression se produisait simultanément (Gilbert et Solter, 1985) et avec la même intensité (West et al., 1986) dans la composante génomique maternelle et la composante génomique paternelle.

La comparaison, aux stades préimplantatoires des profils de synthèse protéique d'embryons androgénotes et gynogénotes (ou parthénotes) n'a, à ce jour, pas permis de mettre en évidence des différences marquées d'expression 
entre le génome paternel et le génome maternel. Au stade 1 cellule, les protéines séquestrées dans chacun des deux pro-noyaux, probablement à partir du cytoplasme de l'œuf, ont le même profil de migration après électrophorèse à 1 dimension (Howlett et al., 1988). Au cours des divisions de segmentation, on observe seulement un niveau général de synthèse plus faible chez les embryons parthénotes (Petzoldt et Hoppe, 1980 ; Barra et Renard, 1988). Dès ces premiers stades de division, le génome paternel intervient dans les mécanismes qui régulent le développement: il contrôle la production de particules de type retroviral (particules $A$ par exemple), produites à partir d'une famille de gènes dispersé dans le génome (Szöllösi et Yotsuyanagi, 1985).

Des différences avec le génome maternel apparaissent si on considère l'expression de gènes situés sur certains chromosomes (ou régions chromosomiques). C'est le cas pour des gènes liés au chromosome $X$. Ainsi le gène qui code pour la phosphoglycérate kinase s'exprime d'abord à partir de la forme allélique maternelle, au $4^{\mathrm{e}}$ jour après la fécondation, puis de la forme paternelle, au $6^{\mathrm{e}}$ jour (Papaioannou et al., 1981 ; Krietsch et al., 1982). Cette différence temporelle d'expression traduit l'existence d'un comportement particulier du chromosome $X$ paternel $(X p)$ : celui-ci est inactivé préférentiellement dans les tissus extraembryonnaires, pendant la formation du trophoblaste et de l'endoderme primitif (Chandra et Brown, 1975 ; Lyon et Rastan, 1984), alors que dans l'ectoderme primitif de l'embryon, l'inactivation concerne indifféremment le chromosome Xp ou sa contrepartie maternelle $\mathrm{Xm}$ à partir du jour 6 (Takagi et Sasaki, 1975 ; West et al., 1977; Harper et al., 1982).

Mais d'autres chromosomes sont concernés par une expression particulière du génome paternel. C'est ce qui ressort indirectement d'études génétiques utilisant des lignées mutantes porteuses de délétions ou de translocations partielles qui, associées à une non-disjonction de chromosomes, aboutissent à la formation de gamètes dans lesquels un chromosome, ou une partie de ce chromosome qui provient d'un seul parent, est absente ou au contraire en double exemplaire (Searle et Beechey, 1985) : I'accouplement de ces souris aboutit à la formation d'embryons dont le génome, tout en gardant une constitution chromosomique équilibrée, a certaines de ses parties qui sont dérivées d'un seul parent.

Ces études démontrent que la duplication dans le génome paternel de la région distale du chromosome 7 ne permet pas de compléter la déficience correspondante du génome maternel, alors que la combinaison réciproque (présence en double exemplaire chez la femelle, et absence chez le mâle) donne des jeunes viables. Inversement, la duplication, dans le génome paternel de régions spécifiques des chromosomes $2,6,8$, associée à leur absence dans le génome maternel, donne des jeunes viables, alors que la combinaison réciproque compromet le développement (Cattanach et Kirk, 1985). Selon la duplication concernée, la mortalité se produit à différents moments de la vie embryonnaire ; elle peut survenir tardivement, par exemple au moment de la naissance dans le cas de non-complémentation de la partie distale du chromosome 2 , ou aboutir à la naissance de jeunes viables, mais de phénotypes différents (Cattanach et Kirk, 1985). Par contre, aucune différence entre les génomes paternels et maternels 
n'est mise en évidence quand la duplication concerne les chromosomes 1, 4, 5, $9,13,14$ et 15 .

Une différence d'expression entre le génome paternel et le génome maternel a pu également être mise en évidence dans une lignée mutante de souris, la lignée Thp ( $T$ hairpin) qui possède un gène muté porté par le chromosome 17 ; cette mutation se traduit par une léthalité de l'embryon quand elle est transmise par le génome maternel (McGrath et Solter, 1984b) alors que la fécondité des accouplements est normale quand elle est transmise par le père.

Enfin on a décrit une lignée de souris, la lignée DDK qui présente une propriété remarquable du point de vue du développement embryonnaire: les femelles de cette lignée accouplées avec des mâles étrangers ont une fertilité diminuée voire complètement abolie, alors qu'avec les croisements réciproques, mâles DDK-femelles étrangères à la lignée, ou avec les croisements à l'intérieur de la lignée, la fertilité est normale (Wakasugi, 1974 ; Wakasugi et Morita, 1977). In vitro, la mortalité apparaît aux environs du stade blastocyste: le début du développement est en apparence normal, mais le trophoblaste ne se forme pas complètement; le phénomène de mortalité embryonnaire n'apparaît que lorsque le noyau paternel d'une autre lignée et le cytoplasme DDK sont mis en présence l'un de l'autre (Renard et Babinet, 1986).

Deux conclusions peuvent être dégagées de ces études. Tout d'abord, il apparaît que les différences d'expression révélées entre les génomes paternels et maternels portent sur l'état de fonctionnement d'une partie de leurs chromosomes et non sur leur constitution génétique qui est la même dans les deux sexes. Dans le cas du chromosome $X$ inactivé préférentiellement dans le trophoectoderme, le chromosome $X p$ peut, dans ces mêmes cellules rester actif si il est présent au sein d'un génome Xp0 (Frels et Chapman, 1980 ; Papaioannou et al., 1981) ; d'autre part, l'inactivation $\mathrm{du} X \mathrm{Xm}$ se produit (quoique avec une fréquence variable) dans les dérivés du trophoblaste d'embryons parthénotes (Endo et Takagi, 1981).

L'inactivation ne résulte donc ni d'un défaut intrinsèque des possibilités de fonctionnement du chromosome, ni de mécanismes d'inactivation particuliers pour les différents lignages cellulaires : elle traduit l'existence d'un " marquage » de ce chromosome, c'est-à-dire d'un comportement différent selon son origine parentale (Chandra et Brown, 1975). Le marquage peut aussi concerner des séquences de DNA exogènes introduites dans l'œuf de souris (transgènes); ces séquences ne s'intègrent pas au hasard dans le génome, et leur expression peut être aisément repérée puisqu'elles ne sont pas présentes dans l'animal hôte : or récemment on a montré, avec différents transgènes, qu'il pouvait dans certains cas, n'y avoir expression que lorsque la transmission s'effectuait par le génome paternel (Swain et al., 1987 ; Hadchouell et al., 1987).

Les études génétiques montrent aussi que pour un gène donné, la présence de 2 copies provenant de l'un ou l'autre des deux parents peut profondément modifier le développement et produire des effets opposés (Cattanach et Kirk, 1985). On peut interpréter ces expériences en considérant que le " marquage » parental intervient en contrôlant le niveau d'expression des gènes et donc que des mécanismes de régulations sont impliqués. Dans les études qui utilisent la migration électrophorétique des produits de ces gènes, ou qui comparent leur 
niveau d'expression, dans chacun des génomes, on ne peut déceler que des gènes dont l'activité est élevée et qui correspondent le plus souvent aux gènes de structure qui s'expriment dans les cellules. Ces approches ne permettent pas d'accéder à l'étude des gènes de régulation dont les produits sont présents en faible quantité, et dont on estime qu'ils ne représentent que $10 \%$ seulement du total des gènes utilisés au cours du développement précoce (Davidson, 1986).

\section{L'action du cytoplasme sur le génome paternel.}

Après fusion entre les membranes du spermatozoïde et de l'ovocyte, la décondensation rapide de la chromatine provoque une augmentation du volume du noyau paternel dont la membrane est dispersée, puis remplacée par une membrane nouvelle qui délimite le pronoyau mâle (McGaughey et Chang, 1969 ; Stefanini et al., 1969). Au bout d'une douzaine d'heures, la chromatine se condense et forme après disparition de la membrane nucléaire les chromosomes paternels.

Le cytoplasme de l'ovocyte régule étroitement l'ensemble des transformations qui aboutissent à la formation du pronoyau paternel : la condensation des chromosomes paternels ne peut plus être obtenue dans un cytoplasme d'ovocytes préalablement activés (Clarke et Masui, 1986) et après fécondation polyspermique seul un nombre limité de noyaux de spermatozoïdes (3 à 4) peut se transformer en pronoyaux mâles (Witowska, 1981 ; Clarke et Masui, 1986).

Plusieurs faits mettent en évidence l'action du cytoplasme sur l'évolution du pronoyau mâle et suggèrent que son activité est régulée différemment de celle du pronoyau femelle. Tout d'abord, au stade 1 cellule, la réplication du DNA commence et se termine plus tôt pour le génome paternel que pour le génome maternel (Luthardt et Donahue, 1973); or à ce stade, le cytoplasme de l'œuf contrôle plus étroitement qu'aux stades ultérieurs le déroulement de la réplication (Howlett, 1986), probablement en permettant au DNA d'accéder à des composants (protéines régulatrices, histones) qui assurent la reprogrammation de la chromatine dont l'activité transcriptionnelle est encore très réduite (Clegg et Piko, 1983). Ensuite, si au stade 1 cellule on réduit d'environ $50 \%$ le volume de cytoplasme d'embryons androgénotes on augmente leurs possibilités de développement de façon plus importante que pour les embryons parthénotes : près de $25 \%$ des androgénotes compactent alors que ce stade n'est pas atteint quand le volume de l'œuf n'est pas diminué. La compaction tend à se produire plus précocement qu'avec les embryons parthénotes (Solter, 1988) ce qui suggère l'existence d'une différence dans la titration des facteurs cytoplasmiques qui seraient impliqués pour la réalisation de cette étape du développement (Levy et al., 1986).

Enfin en utilisant les embryons de la lignée DDK (voir ci-dessus), nous avons mis directement en évidence l'existence d'une action spécifique du cytoplasme de l'œuf sur le noyau paternel. En effet, si on introduit dans un œuf BALB/c fécondé par un mâle BALB/c un peu de cytoplasme d'un œuf DDK (fécondé ou non), on obtient un arrêt du développement aux environs du stade blastocyste ; par contre, 
la survie est normale si on effectue la même opération avec un œuf BALB/c fécondé par un spermatozoïde DDK (fig. 2). II y a donc bien interaction entre le génome paternel et le cytoplasme environnant avec manifestation d'une incompatibilité quand le génome paternel n'est pas de type DDK. La même opération effectuée au stade 2 cellules, donne des résultats identiques : après l'addition de cytoplasme DDK, l'arrêt du développement ne se produit que lorsque les noyaux de l'embryon sont issus d'un œuf fécondé par un mâle non DDK (Renard et al., à paraître).

\section{$B A L B / C-B A L B / C \quad D D K-D D K \quad B A L B / C-D D K$}

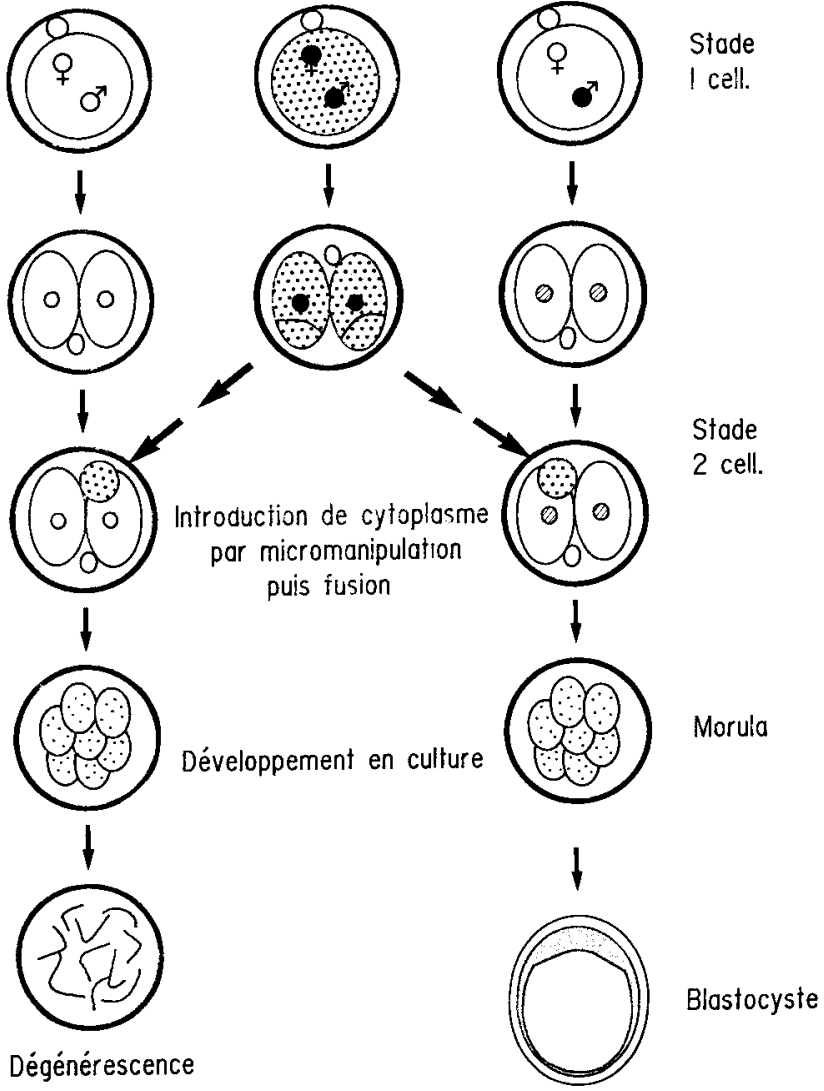

FIG. 2. - Mise en évidence, à partir des 2 lignées de souris $D D K(\bullet)$ et $B A L B / c(O)$ d'une action du cytoplasme sur la composante paternelle du génome de l'embryon (Renard et al., à paraître).

Le cytoplasme peut donc agir spécifiquement sur la composante paternelle d'un génome diploïde déjà activé. Ces données jointes au fait qu'il existe une 
ségrégation de la chromatine selon l'origine parentale dans les noyaux en interphase de l'embryon au cours des premiers clivages (Odartchenko et Kenelis, 1973 ) indiquent bien que le cytoplasme peut agir différemment sur chacune des deux composantes parentales au début du développement.

Inversement, le génome paternel peut modifier les caractéristiques du cytoplasme. En effet, nous avons montré que le cytoplasme d'un ovocyte DDK énucléé après avoir été fécondé par un spermatozoïde non DDK est moins capable d'assurer le développement normal d'une combinaison génomique diploïde viable, que le cytoplasme DDK après fécondation par un spermatozoïde lui-même DDK (Renard et Babinet, 1986). Ce résultat met clairement en évidence un effet très précoce du génome paternel sur le développement des embryons, et nous pensons qu'il révèle un événement jusqu'alors passé inaperçu car se déroulant normalement avec les souches non DDK.

\section{Conclusion.}

Les données présentées ci-dessus, montrent que la présence d'un génome paternel est nécessaire pour assurer un développement normal, et que son action au cours du développement est différente de celle du génome maternel. Des interactions entre chromosomes homologues jouent donc un rôle important dans l'embryogenèse: elles traduisent l'existence d'un marquage spécifique qui dépend de l'origine parentale. Ce marquage doit être réversible à chaque génération puisque le génome est transmis indifféremment dans les deux sexes.

Sa nature moléculaire n'est pas connue actuellement; elle dépend probablement des réorganisations de la chromatine du noyau dans les cellules germinales pendant la gamétogenèse (Groudine et Conkin, 1985). Celle-ci acquiert une structure particulière notamment au cours de la spermiogenèse, lors des transformations qui permettent le conditionnement du DNA dans le spermatozoïde (Balhorn, 1982). Ces remaniements comprennent le remplacement des histones par des protéines basiques de petit poids moléculaire riches en arginine, la condensation du DNA par la formation de ponts disulfures, et la décroissance concomitante du volume du noyau (Fawcett et al., 1971 ; Calvin et Bedford, 1971). L'expression génique au cours de la phase haploïde qui est importante, contrairement à ce qui se passe au cours de l'ovogenèse, intervient dans ces remaniements (Willison et Ashworth, 1987; Hecht, 1986).

La méthylation des gènes pourrait également jouer un rôle. Plusieurs observations faites à partir de gènes endogènes ou de transgènes, montrent que le degré de méthylation peut être très différent selon la lignée germinale (Sandford et al., 1987 ; revues dans Surani, 1986 ; Solter, 1988).

Ces résultats, obtenus principalement chez la souris, apportent un éclairage nouveau à des situations jusqu'alors peu comprises, rencontrées chez l'homme. Des maladies génétiques, comme la maladie de Huntington, et l'Ataxia Cerebella, surviennent plus précocement quand elles sont transmises par le père, et l'existence d'un effet de marquage des chromosomes paternels est envisagé (Erickson, 1985 ; Harding, 1981). Certaines formes de stérilité idiopathique, qui 
représente une proportion importante des cas de stérilité, environ $20 \%$ (Spira et de Mouzon, 1985) pourraient également relever du marquage différentiel des chromosomes parentaux.

$27^{e}$ Réunion de la Société francaise pour l'Etude de la Fertilité Paris, 29, 30 sept., $1^{\text {er }}$ oct 1988.

Remerciements. - Ce travail a été réalisé dans le cadre d'un contrat INSERM (CRF-864002) sur l'«Etude de la contribution respective des génomes mâles et femelles dans le développement de l'embryon de souris ». J. P. Renard est affilié à l'INRA.

\section{Références}

ANDEREGG C., MARKERT C. L., 1987. Successful rescue of microsurgically produced homozygous uniparental mouse embryos via production of aggregation chimeras. Proc. nat. Acad. Sci. USA, 83, 6509-6513.

BALHORN R., 1982. A model for the studies of chromatin in mammalian sperm. J. Cell Biol., 93, 298-305.

BARRA J., RENARD J.-P., 1988 . Diploid mouse embryos constructed at the late 2-cell stage from haploid parthenotes and androgenotes can develop to term. Development, 102, 773-779.

BARTON S. C., SURANI M. A. H., NORRIS M. L., 1984. Role of paternal and maternal genomes in mouse mouse development. Nature, 311, 374-376.

BEARDEN H. J., HANSEL W. M., BRATTEN W., 1956. Fertilization and embryonic mortality rates of bulls with histories of either low or high fertility in artificial breeding. J. Dairy Sci., 39 , 312-318.

BENSAUDE O., BABINET C., MORANGE M., JACOB F., 1983. Heat shock proteins, first major products of zygotic gene activity in mouse embryo. Nature, 305, 331-333.

BRAUDE P., BOLTON V., MOORE S., 1988. Human gene expression first occurs between the four and eight-cell stages of preimplantation development. Nature (Lond.), 232, 459-461.

BRINSTER R. L., 1973. Parental glucose phosphate isomerase activity in three-day mouse embryos. Biochem. Genet., 9, 187-191.

CALVIN H. L., BEDFORD J. M., 1971. Formation of disulfide bonds in the nucleus and accessing structures of mammalian spermatozoa during maturation in the epididymis. J. Reprod. Fert., (suppl.) 13, 65-75.

CATTANACH B. M., KIRK M., 1985. Differential activity of maternally and paternally derived chromosome regions in mice. Nature, 315, 496-498.

CHANDRA H. S., BROWN S. W., 1975. Chromosome imprinting and the mammalian X chromosome. Nature, 53, 165-168.

CLARKE H. J., MASUI Y., 1986. Transformation of sperm nuclei to metaphase chromosomes in the cytoplasm of maturing oocytes of the mouse. J. Cell Biol., 102, 1039-1044.

CLEGG K. B., PIKO L., 1983. Quantitative aspects of RNA synthesis and polyadenylation in one cell and two cell mouse embryos. J. Embryol. exp. Morphol., 74, 169-182.

CUELLAR O., 1977. Animals parthenogenesis. Science, 197, 837-843.

DAVIDSON E. H., 1986. From genome to embryo: the regulation of gene activity in early development, 2-44. In DAVIDSON E. H., Gene activity in early development. 3rd Ed. Acad. Press.

ENDO S., TAKAGI N., 1981. A preliminary cytogenetic study of $X$ chromosome inactivation in diploid parthenogenetic embryos from LT/SV mice. Jpn. J. Genet., 56, 349-356.

ERICKSON R. P., 1985. Chromosomal imprinting and the parent transmission specifique variation in expressivity of huntington disease. Am. J. Hum. Genet., 37, 827-829.

FAWCETT D. W., ANDERSON W. A., PHILIPS D. W., 1971. Morphogenetic factors influencing the shape of the sperm head. Dev. Biol., 26, 220-251. 
FRELS W. I., CHAPMAN V. M., 1980. Expression of the maternally derived $X$ chromosome in the mural trophoblast of the mouse. J. Embryol. exp. Morph., 56, 179-180.

GILBERT S. F., SOLTER D., 1985. Onset of paternal and maternal GPi-1 expression in preimplantation mouse embryos. Dev. Biol., 109, 515-517.

GROPP A., WIKING H., 1981. Robertsonian translocations : cytology, meiosis, segregation patterns and biological consequences of heterozygosity. In R. J. PERRY, Biology of the house mouse. Symp. Zool. Soc. Lond., 47, 141-181.

GROUDINE M., CONKIN K. F., 1985. Chromatine structure and de novo methylation of sperm DNA : implications for activation of the paternal genome. Science, 236, 962-964.

GUSTAVSSON I., 1979. Distribution and effects of 1-29 Robertsonian translocation in cattle. $J$. Dairy Sci., 62, 825-835.

HADCHOUELL M., FARZA H., SIMON D., TIOLLAIS P., POURCEL C., 1987. Maternal inhibition of hepatitis B surface antigen gene expression in transgenic mice correlates with de novo methylation. Nature (Lond.). 329, 454-456.

HARDING A. E., 1981. Genetic aspects of autosomal dominant late onset cerebellar atasia. J. Med. Genet., 18, 436-441

HARPER M. L., FOSTEN M., MONK M., 1982. Preferential paternal X inactivation in extraembryonic tissues of early mouse embryos. J. Embryol. exp. Morph., 67, 127-135.

HECHT N. B., 1986. Regulation of gene expression during mammalian spermatogenesis, 151-193. In ROSSANT J., PEDERSEN R. A., Experimental approaches to mammalian embryonic deve/opment. Cambridge Univ. Press.

HOWLETT S. K., 1986. The effect of inhibiting DNA replication in the one cell mouse embryo. Roux's Arch. Dev. Biol., 195, 499-505.

HOWLETT S. K., BARTON C., NORRIS M. L., SURANI M. A. H., 1988 . Nuclear and cytoplasmic localization of newly synthetized protein in the early mouse embryo. Development, 103. $129-134$

KAUFMAN M. H., 1983. Early mammalian development: parthenogenetic studies. In BARLOW P. W., GREEN P. B., WYLIE C. C., Developmental and cell biology series. Cambridge Univ. Press.

KRIETSCH W. K. G., FUNDELE R., KUNTZ G. W. F., FEHLAU M., BURKI K., ILLMENSEE K. 1982. The expression of $X$-linked phosphoglycerate kinase in the early mouse embryo Differentiation, 23, $141-144$.

LEVY J. B., JOHNSON M. H., GOODALL H., MARO B., 1986. The timing of compaction : control of a major developmental transition in mouse early embryogenesis. J. Embryol. exp. Morphol., 95, 213-237.

LUTHARDT F. W., DONAHUE R. P., 1973. Pronuclear DNA synthesis in mouse eggs. Exp. Cel/ Res., 82, 143-151.

LYON M. F., RASTAN S., 1984. Parental source of chromosome imprinting and its relevance for $X$ chromosome inactivation. Differentiation, 26, 63-67.

MANN J. R., LOVELL-BADGE R. H., 1984. Inviability of parthenogenones is determined by pronuclei, not egg cytoplasm. Nature, 310, 66-67.

MATTEI J. F., 1984. Influence of the male on embryonic mortality, 350-369. In COUROT M., The male in reproduction. M. Nijhoff, the Hague.

McGAUGHEY R.W., CHANG M. C., 1969. Meiosis of mouse eggs: before and after sperm penetration. J. exp. Zool, 170, 397-410.

McGRATH J., SOLTER D., 1983. Nuclear transplantation in the mouse embryo by microsurgery and cell fusion. Science, 220, 1300-1302.

McGRATH J., SOLTER D., 1984a. Mouse embryogenesis requires both the maternal and paternal genomes. Cell, 37, 179-183.

McGRATH J., SOLTER D., 1984b. Maternal thp lethality in the mouse is a nuclear, not cytoplasmic, defect. Nature, 308, 550-551.

NAGY A., PALDI A., DEZSO L., VARGA L., MAGYAR A., 1987. Prenatal fate of parthenogenetic cells in mouse aggregation chimaeras. Development, 101, 67-71.

ODARTCHENKO N., KENELIS T., 1973. Localization of paternal DNA in interphase nucleus of mouse eggs during early cleavage. Nature, 241, 528-529. 
PAPAIOANNOU V. E., WEST J. D., BUCHER T., LINKE I. M., 1981. Non random X-chromosome expression early in mouse development. Develop. Genet., 2, 305-315.

PETZOLDT U., HOPPE P. C., 1980. Spontaneous parthenogenesis in Mus Musculus : comparison of protein synthesis in parthenogenetic and normal preimplantation embryos. Mol. gen. Genet., 180, 547-552.

RENARD J.-P., BABINET C., 1986. Identification of a paternal developmental effect on the cytoplasm of one-cell-stage mouse embryos. Proc. nat. Acad. Sci. USA, 83, 6883-6886.

SANDFORD J. P., CLARK H. J., CHAPMAN V. M., ROSSANT J., 1987. Differences in DNA methylation during oogenesis and spermatogenesis and their persistence during early embryogenesis. Genes Develop., 1, 1039-1046.

SAWICKI J. P., MAGNUSSON T., EPSTEIN C. J., 1981. Evidence for the expression of paternal genome in the two-cell stage mouse embryo. Nature, 294, 450-451.

SEARLE A. G., BEECHEY C. V., 1985. Non complementation phenomena and their bearing on non disjunctional effects, 363-376. In DELLARCO V. L., VOYTEK P. E., HOLLUENDER A., Aneuploidy. Plenum Press, New York.

SOLTER D., 1988. Differential imprinting and expression of maternal and paternal genomes. Ann. Rev. Gen. (in press).

SPIRA A., de MOUZON J., 1985. Infécondité idiopathique: $18 \%$ des couples infertiles. Inserm Symp., 24, 263-275.

STEFANINI M., OURA C., ZAMBONI L., 1969. Ultrastructure of fertilization in the mouse. 2. Penetrat ion of sperm into ovum. J. Submicrosc. Cytol., 1. 1-23.

STEVENS L. C., 1978. Totipotent cells of parthenogenetic origin in chimaeric mouse. Nature, 276 , 266-267.

STEVENS L. C., VARNUM D. S., EICHER E. M., 1977. Viable chimeraes produced from normal and parthenogene. Nature, 269, 515-517.

SURANI M. A. H., 1986. Evidences and consequences of differences between maternal and paternal genomes during embryogenesis in the mouse, 401 -434. In ROSSANT J., PEDERSEN R. A., Experimental approaches to mammalian embryonic development. Cambridge Univ. Press.

SURANI M. A. H., BARTON S. C., KAUFMAN M. H., 1977 . Development to term of chimaeras between diploid parthenogenetic and fertilized embryos. Nature, 270, 601-603.

SURANI M. A. H., BARTON S. C., NORRIS M. L., 1984. Development of reconstituted mouse eggs suggest imprinting of the genome during gametogenesis. Nature, 308, 548-550.

SURANI M. A. H., BARTON S. C., NORRIS M. L., 1986. Nuclear transplantation in the mouse: Heritable differences between parental genomes after activation of the embryonic genome. Cell, 45, 127-136.

SURANI M. A. H., BARTON S. C., NORRIS M. L., 1987. Influence of parental chromosomes on spatial specificity in androgenetic parthenogenetic chimaeras in the mouse. Nature, 326. 395-397.

SURANI M. A. H., BARTON S. C., HOWLETT S. K., NORRIS M., 1988. Influence of chromosomal determinants on development of androgenetic and parthenogenetic cells. Development, 103, $171-178$.

SWAIN J. L., STEWART T. A., LEDER P., 1987. Parental legacy determines methylation and expression of an autosomal transgene : a molecular mechanism for parental imprinting. Cell, 50, 719-727.

SZÖLLÖSI D., YOTSUYANAGI Y., 1985. Activation of paternally derived regulatory mechanism. Dev. Biol., 111, 256-259.

SZULMAN A. E., SURTI V., 1984. Complete and partial hydatiform moles: cytogenetic and morphological aspects, 135-146. In PATILLO R. A., HUSSA R. O., Human trophoblast and neoplasms. Plenum Press, New York.

TAKAGI N., SASAKI M., 1975. Preferential inactivation of the paternally derived X-chromosome in the extraembryonic membranes of the mouse. Nature, 256, 640-642.

THIBAULT C., 1985. Rôle du gamète mâle dans le développement embryonnaire chez les mammifères. $2^{e}$ Jour. Fed. nat. Col. Gyn. Med., 17-24.

WAKASUGI N., 1974. A genetically determinant incompatibility system between spermatozoa and eggs leading to embryonic death in mice. J. Reprod. Fertil., 41, 85-96. 
WAKASUGI N., MORITA M., 1977. Studies of the development of F1 embryos from inter-strain crosses involving DDK mice. J. Embryol. exp. Morph., 38, 211-216.

WEST J. D., FRELS W. I., CHAPMAN V. M., PAPAIANOU V. E., 1977. Preferential expression of the maternally derived $X$ chromosome in the mouse yolk sac. Cell, 12, 873-882.

WEST J. D., LEASK R., GREEN F., 1986. Quantification of the transition from oocyte-coded to embryo-coded glucose phosphate isomerase in mouse embryos. J. Embryol. exp. Morph., 97, 225-237.

WILLISON K., ASHWORTH A., 1987. Mammalian spermatogenic gene expression. Trends Gen., 3, $351-355$.

WITOWSKA A., 1981. Pronuclear development and the first cleavage division in polyspermic mouse eggs. J. Reprod. Fertil., 62, 493-498.

WUDL L., CHAPMAN V., 1976. The expression of glucuronidase during preimplantation development of mouse embryos. Dev. Biol., 48, 104-109. 